\title{
LINEAR AND NONLINEAR EVOLUTION OF ISOLATED DISTURBANCES IN A GROWING THERMAL BOUNDARY LAYER IN POROUS MEDIA
}

\author{
A. Selim, D.A.S. Rees \\ Department of Mechanical Engineering, University of Bath, Bath BA2 7AY, UK.
}

\begin{abstract}
We consider the onset and development of convection in a saturated porous half-space which is initially cold, but where the lower boundary has its temperature raised suddenly to a new uniform level. The resulting thermal boundary layer diffuses upwards and eventually becomes thermoconvectively unstable. Previous works by the present authors have considered in turn linearised theory, the nonlinear development of cells, and the destabilisation of such cells due to subharmonic disturbances. In all three papers it was assumed that the convection pattern is horizontally periodic. In the present paper we relax this restriction by considering how an isolated disturbance develops in time, and this is compared with the horizontally periodic flows. New cells are generated outboard of existing ones so that the convecting region spreads horizontally with time. The effective wavelengths are also found to increase with time, newer cells having larger wavelengths than older ones. We also consider the nonlinear development of these disturbances.
\end{abstract}

Keywords: Porous media, free convection, unsteady boundary layer, isolated disturbance, linear instability, nonlinear convection.

PACS: $44.25 .+f, 44.30 .+v, 47.11 . B c, 47.15 . \mathrm{Cb}, 47.20 . \mathrm{Bp}, 47.56 .+\mathrm{r}$

\section{INTRODUCTION}

The effect of suddenly changing the temperature of a horizontal boundary of a porous half-space has studied by many researchers. The detailed history of this topic and the methods used to determine the stability characteristics of the resulting thermal boundary layer is too long to be repeated here, but a recent summary may be found in [4].

Within the context of linear stability theory, disturbances are usually Fourier-decomposed into their separate wavenumbers. The resulting set of equations for each monochromatic mode is a parabolic partial differential system which may be solved numerically. Many authors have approximated this system by reducing it ordinary differential eigenvalue form where the eigenvalue is the critical time. The computed critical times then depend strongly on how the time-deriviative is approximated - see Table 1 in [4]. Other methods, such as energy stability analyses and local Rayleigh number analyses also provide their own set of onset data; see [4].

This lack of mathematical consistency that motivated Selim and Rees [1] to solve the full parabolic system numerically. Although they showed that disturbances which are introduced very early tend to- wards a unique evolving profile, it was found that the magnitude of the evolving disturbance depends on how that magnitude is measured. Thus an energy functional, the surface rate of heat transfer, and the maximum temperature all give different neutral curves. Of these, the energy functional yields the largest region of instability and the earliest onset time. These differences are due to the fact that the disturbance profile varies as it evolves.

In this paper we consider how an isolated disturbance evolves in time, both within the linear regime and when it is nonlinear. The full disturbance equations are solved numerically, and our results interpreted in the light of the monochomatic behaviour described in [1].

\section{NOMENCLATURE}

$A=$ amplitude of disturbance

$g=$ gravity

$k=$ disturbance wavenumber

$K=$ permeability

$L=$ length scale

$N=$ nonlinear terms

$p=$ pressure

$Q=$ surface rate of heat transfer 
$u, v=$ horizontal and vertical velocities

$t=$ time

$T_{w}=$ dimensional wall temperature

$T_{\infty}=$ dimensional ambient temperature

$x, y=$ horizontal and vertical coordinates

Greek symbols

$\alpha=$ thermal diffusivity

$\beta=$ expansion coefficient

$\eta=$ similarity variable

$\theta, \Theta=$ temperature

$\mu=$ dynamic viscosity

$\rho=$ density

$\tau=$ scaled time

$\psi, \Psi=$ streamfunction

Subscripts and superscripts

${ }^{\prime}=$ derivative with respect to $\eta$

$\tau=$ derivative with respect to $\tau$

\section{GOVERNING EQUATIONS}

We are considering the instability of an initially quiescent semi-infinite region of saturated porous medium at the uniform temperature, $T_{\infty}$, the lower boundary of which has its temperature raised suddenly to the new value, $T_{w}$. The porous medium is homogeneous and isotropic, and the flow is governed by Darcy's law modified by the presence of buoyancy and subject to the Boussinesq approximation. The governing nondimensional equations are,

$$
\begin{gathered}
\frac{\partial u}{\partial x}+\frac{\partial v}{\partial y}=0 \\
u=-\frac{\partial p}{\partial x}, \quad v=-\frac{\partial p}{\partial y}+\theta \\
\frac{\partial \theta}{\partial t}+u \frac{\partial \theta}{\partial x}+v \frac{\partial \theta}{\partial y}=\frac{\partial^{2} \theta}{\partial x^{2}}+\frac{\partial^{2} \theta}{\partial y^{2}} .
\end{gathered}
$$

The appropriate boundary conditions are that,

$$
\begin{aligned}
y=0: & v=0, \quad \theta=1 \\
y \rightarrow \infty: & v, \theta \rightarrow 0,
\end{aligned}
$$

while $\theta=0$ everywhere for $t<0$.

The pressure may be eliminated by introducing the streamfunction, $\psi$, according to,

$$
u=-\frac{\partial \psi}{\partial y} \quad \text { and } \quad v=\frac{\partial \psi}{\partial x},
$$

and therefore Eq. (1a), is automatically satisfied. The full nonlinear equations now take the form,

$$
\frac{\partial^{2} \psi}{\partial x^{2}}+\frac{\partial^{2} \psi}{\partial y^{2}}=\frac{\partial \theta}{\partial x}
$$

$$
\frac{\partial \theta}{\partial t}+\frac{\partial \psi}{\partial x} \frac{\partial \theta}{\partial y}-\frac{\partial \psi}{\partial y} \frac{\partial \theta}{\partial x}=\frac{\partial^{2} \theta}{\partial x^{2}}+\frac{\partial^{2} \theta}{\partial y^{2}} .
$$

The streamfunction is taken to be zero on $y=0$ and as $y \rightarrow \infty$. We note that there is no Darcy-Rayleigh number in the above; this is a natural consequence of using

$$
L=\frac{\mu \alpha}{\rho g \beta K\left(T_{w}-T_{\infty}\right)}
$$

as a length scale. The basic quiescent state is

$$
\psi=0, \quad \theta=\operatorname{erfc} \eta=\frac{2}{\sqrt{\pi}} \int_{\eta}^{\infty} e^{-\xi^{2}} d \xi
$$

(see [1]) where the similarity variable, $\eta$, is given by

$$
\eta=\frac{y}{2 \sqrt{t}} .
$$

If we replace $\theta$ by $\theta+\operatorname{erfc}(\eta)$, and then introduce (7) and $\tau=\sqrt{t}$ as alternative vertical and time coordinates, then we obtain the full pertubation equations,

$$
4 \tau^{2} \frac{\partial^{2} \psi}{\partial x^{2}}+\frac{\partial^{2} \psi}{\partial \eta^{2}}=4 \tau^{2} \frac{\partial \theta}{\partial x}
$$

$2 \tau \frac{\partial \theta}{\partial \tau}+N(\psi, \theta)=4 \tau^{2} \frac{\partial^{2} \theta}{\partial x^{2}}+\frac{\partial^{2} \theta}{\partial \eta^{2}}+2 \eta \frac{\partial \theta}{\partial \eta}+\frac{4 \tau e^{-\eta^{2}}}{\sqrt{\pi}} \frac{\partial \psi}{\partial x}$

where the nonlinear terms are given by,

$$
N(\psi, \theta)=2 \tau\left(\frac{\partial \psi}{\partial x} \frac{\partial \theta}{\partial \eta}-\frac{\partial \psi}{\partial \eta} \frac{\partial \theta}{\partial x}\right) .
$$

\section{LINEAR THEORY: MONOCHROMATIC MODES}

The analysis of Selim and Rees [1] neglects the nonlinear term in (8) and Fourier-decomposes the disturbances according to

$$
\psi=\Psi(\eta, \tau) \cos k x, \quad \theta=\Theta(\eta, \tau) \sin k x,
$$

where $k$ is the wavenumber. The reduced variables, $\Psi$ and $\Theta$, satisfy,

$$
\begin{gathered}
\Psi^{\prime \prime}-4 \tau^{2} k^{2} \Psi=4 \tau^{2} k \Theta \\
2 \tau \Theta_{\tau}=\Theta^{\prime \prime}+2 \eta \Theta^{\prime}-4 \tau^{2} k^{2} \Theta-\frac{4}{\sqrt{\pi}} \tau k e^{-\eta^{2}} \Psi .
\end{gathered}
$$

Artifical disturbances were placed within the boundary layer and their evolution with time was noted. If we use the surface rate of heat transfer as the monitoring function, then the time at which it ceases to decay and begins to grow is deemed to be the critical time. It was in this manner that the neutral curve which is shown in Fig. 1 was constructed. 


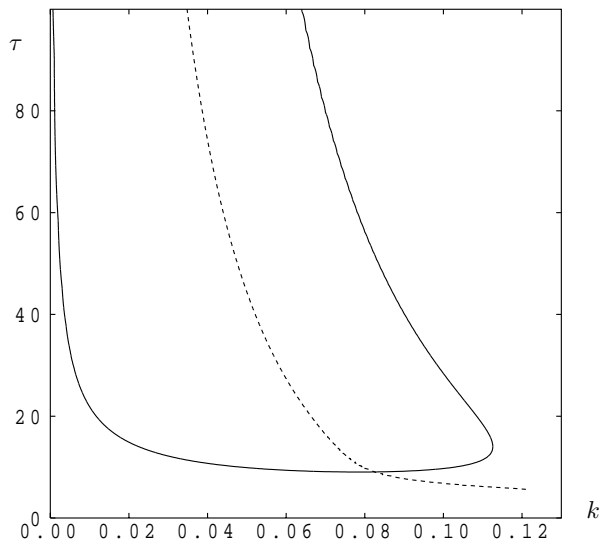

FIGURE 1. Showing the neutral curve (continuous) and the line of fastest disturbance growth (dashed)

Figure 1 also shows the locus of points at which the growth rate of the surface rate of heat transfer has been maximised over all wavenumbers. Thus, as time progresses, and as the basic thermal boundary layer increases in thickness, the most dangerous disturbance corresponds to decreasing values of the wavenumber. This corresponds to increasingly large wavelengths, which is intuitive, given that the boundary layer thickness is increasing.

\section{LINEAR THEORY: LOCALISED DISTURBANCES}

Localised disturbances was modelled by solving Eqs. (8a) and (8b) without the nonlinear terms. Second order accurate central differences in space and the backward Euler method in time were used. This implicit scheme relied on the FAS multigrid method with line relaxation in both directions. An adaptive timestepping strategy was used in order to increase efficiecy. This scheme was first used in Rees and Bassom [5] where more detail may be found, and has been used successfully in [6-8].

The initial disturbance was $\theta=A e^{-x^{2} / 100} \eta e^{-\eta}$, where the value of $A$ is irrelevant for linear theory. The computational domain was $0 \leq x \leq 2000$ and $0 \leq \eta \leq 8$, the latter being well in excess of what was needed to contain the evolving disturbances. The uniform grid used $640 \times 48$ points. The boundary at $x=0$ was an axis of symmetry where $\psi=0$ and $\partial \theta / \partial x=0$. The value, $x=2000$, was just enough to contain the spreading disturbance up to $\tau=100$, and just over 3500 timesteps were used.

Figure 2 shows disturbance isotherms at different stages of the evolution. As time progresses the disturbance also propagates outwards into undisturbed regions by the successive generation of new cells. Such front propagation is well-known in other contexts, such as the Bénard problem (see Getling [9], for example, and the comprehensive review by van Saarloos [10]), but has not been studied in the context of convective instablities in porous media. The main distinguishing features of the cellular patterns shown in Fig. 2 are that new cells are wider that their predecessors and that all cells tend to widen slowly as time progresses. These features are also shown in Fig. 3, which depicts the boundaries between the 'thermal' cells, i.e. those locations at which the surface rate of heat transfer, $Q$, is zero. Thus the addition of new cells may be seen clearly.

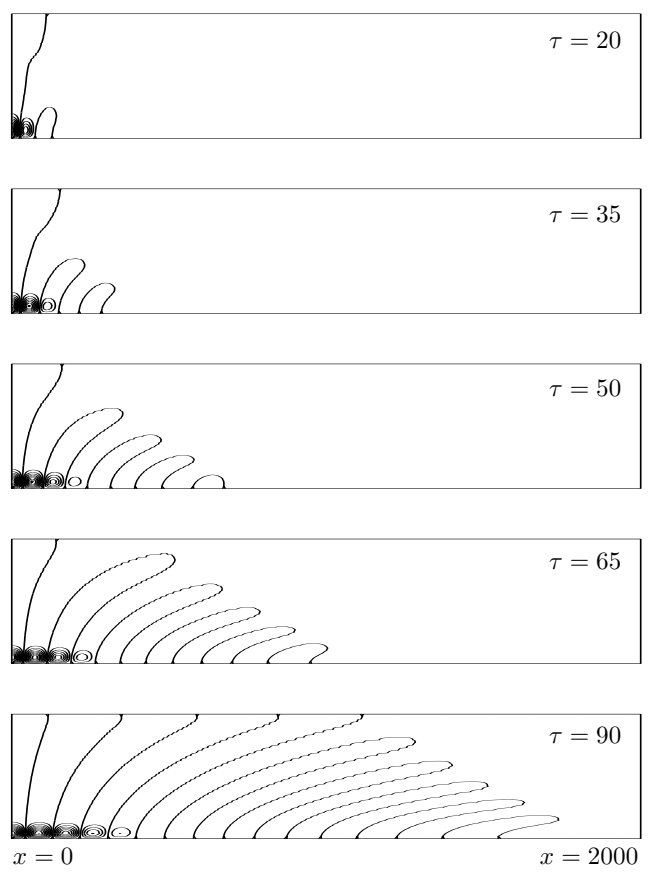

FIGURE 2. Disturbance isotherms at the given times. The upper boundary corresponds to $\eta=8$.

It is well-known that the propagation speed of the disturbance front tends towards a constant value for the analogous Bénard layer [11], and it is of interest to compare this behaviour with the present problem. Figure 3 cannot be used directly because it is plotted in terms of $\tau$, rather than $t$. However, the numerical data shows that the propagation speed is not constant, although the values of $x / t$ which correspond to the last three new cells depicted in Fig. 3, are all approximately equal to 0.23 . In our view, this is insufficient evidence to assert that the propagation speed relaxes to a constant value; further computations in larger domains are required. 


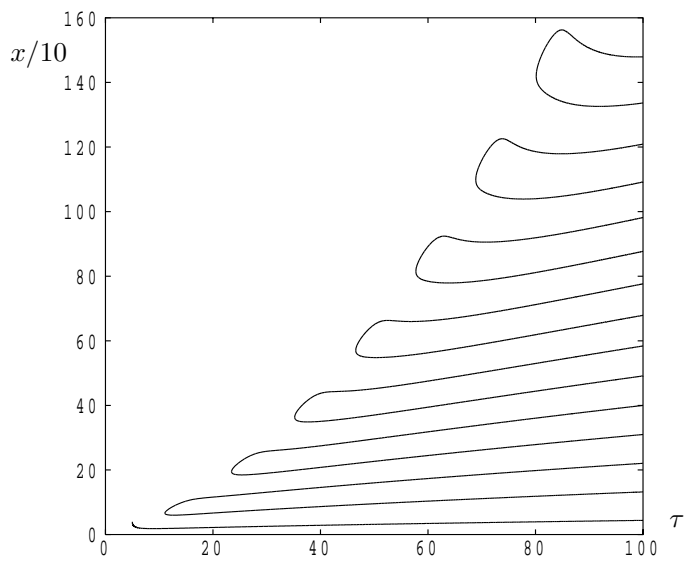

FIGURE 3. Showing the contours of zero rate of heat transfer of the disturbance $(Q=0)$ at the surface.

Of more interest, perhaps, are the abovementioned facts that the width of each new cell increases and that all cells tend to get wider with time. These are consistent with the identity of the fastest growing mode shown in Fig. 1. Therefore we have computed the widths of the cells from the data shown in Fig. 3, and plotted these against $\tau$ in Fig. 4. Given that one cell corresponds to half a wavelength, we have computed the corresponding wavenumber for each 'cell' (using wavenumber $\times$ cell width $=\pi$ ), and these are shown in Fig. 5 .

Figure 4 shows quite clearly that the cell width of each new cell (i.e. the upper curve of each pair) is substantially larger than (i) those that already exist and (ii) the width corresponding to the fastest growing mode. This is not surprising because new cells are unconfined unlike their counterparts in a periodic cellular pattern. However, these cells narrow at first and particularly so when another new cell is generated on their formerly free side. So we see an initial narrowing followed by a slow increase in width, but it is an increase which causes the width gradually approach that of the fastest growing mode as it too changes in time.

The situation as seen from the point of view of the wavenumber is presented in Fig. 5. The wavenumber corresponding to the innermost cell (i.e. the one centred at $x=0$ ) continues to have a value which is slightly larger than that of the fastest growing mode. We think that this is due to the fact that neighbouring cells inhibit the horizontal growth of this cell merely by their presence. But the whole system gradually expands as well as spreading by cell generation as time progresses. The characteristic wavenumber of fastest growing monochomatic mode serves as a reference wavenumber for this pro- cess as time progresses.

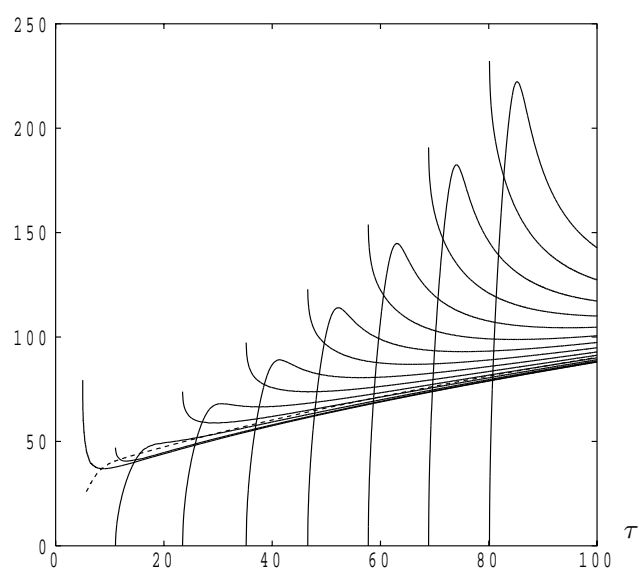

FIGURE 4. Showing the width of the thermal cells as a function of $\tau$ (continuous lines) and the cell width corresponding to the fastest growing wavenumber shown in Fig. 1 (dashed line).

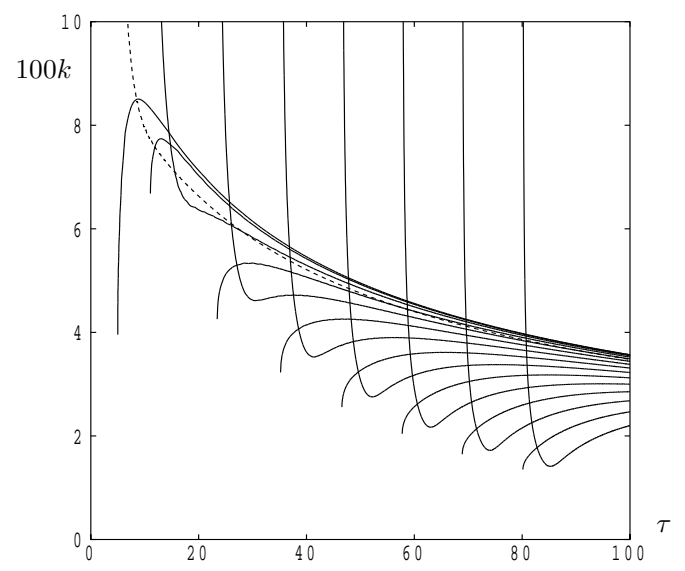

FIGURE 5. As Figure 4, but in terms of equivalent wavenumber.

\section{NONLINEAR COMPUTATIONS}

The same numerical method was used to determine how nonlinear effects alter the pattern of cellular evolution, and these patterns are summarised in Fig. 6. Although one may change the value of $\tau$ at which a disturbance may be introduced, and change the profile of the initial disturbance from that given above, we restrict attention to changing values of $A$.

Figure 6 a corresponds to $A=10^{-10}$. For such a small value, the disturbance remains within the linear regime for most of its evolution. The only distictive modification is that the final cell which appeared at the top of Fig. 2 does not now form. 


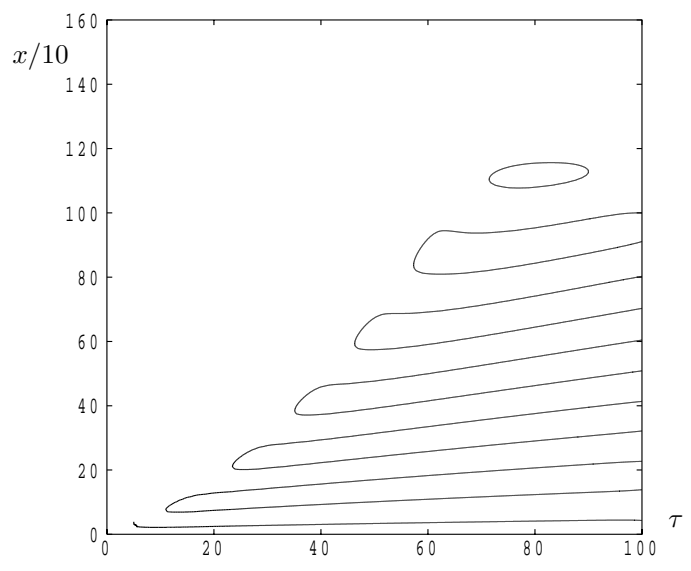

FIGURE 6a. Contours of $Q=0$ when $A=10^{-10}$.

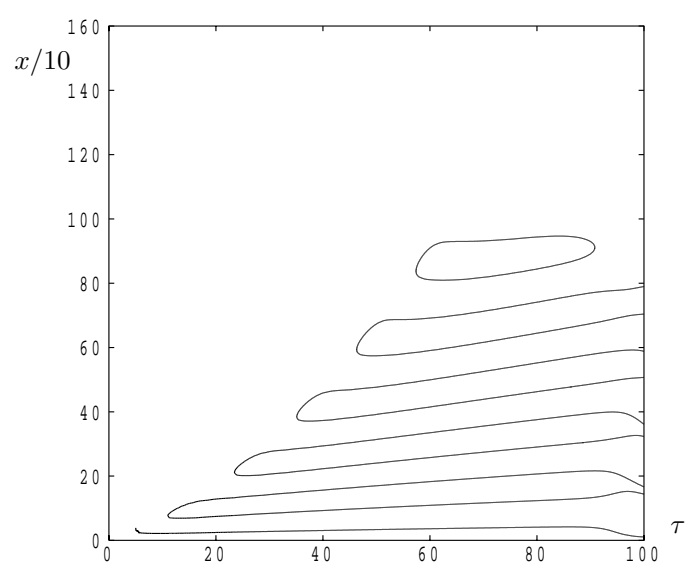

FIGURE 6b. Contours of $Q=0$ when $A=10^{-8}$.

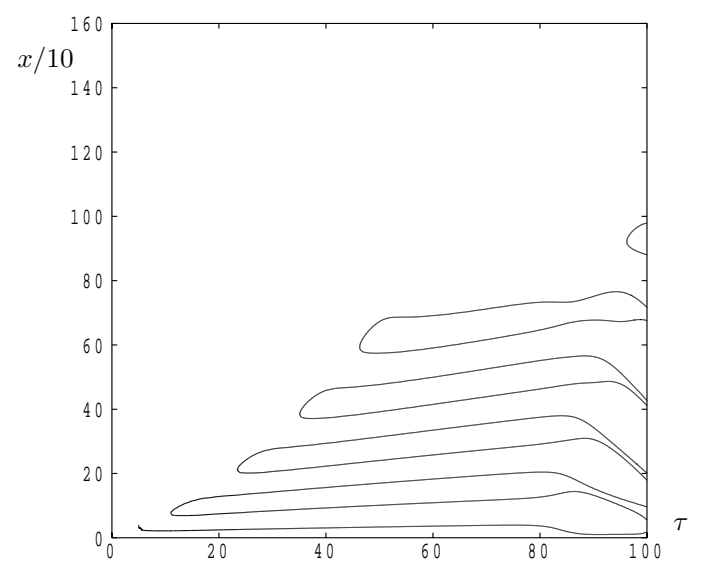

FIGURE 6c. Contours of $Q=0$ when $A=10^{-6}$.

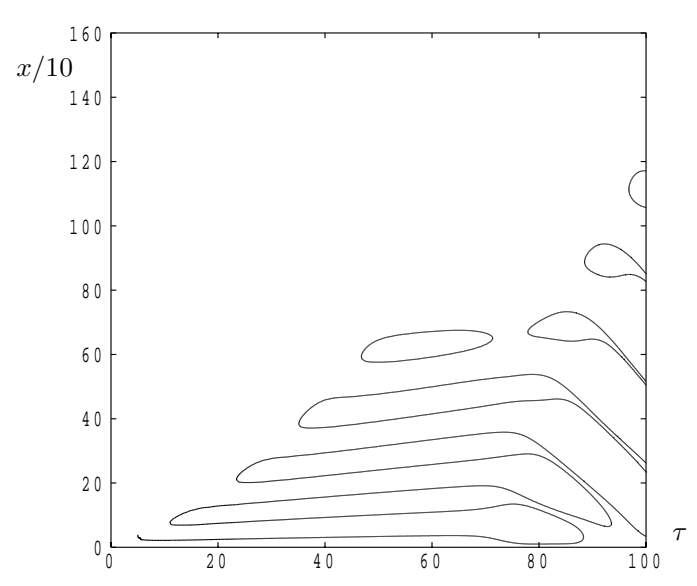

FIGURE 6d. Contours of $Q=0$ when $A=10^{-4}$.

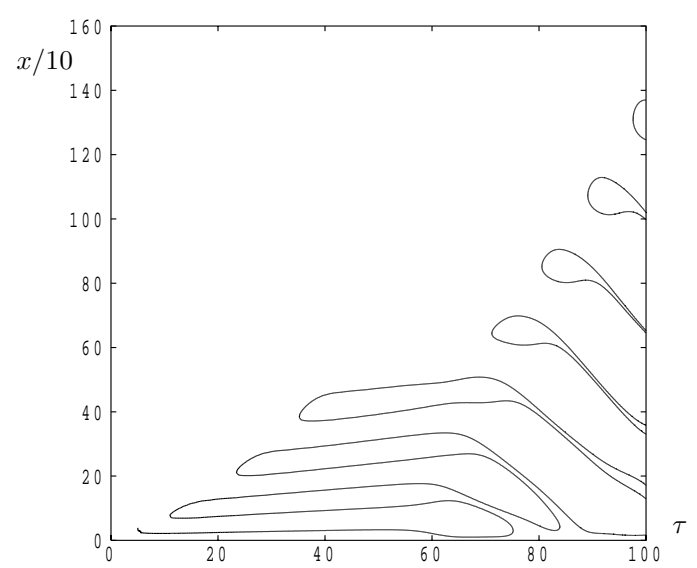

FIGURE 6e. Contours of $Q=0$ when $A=10^{-2}$.

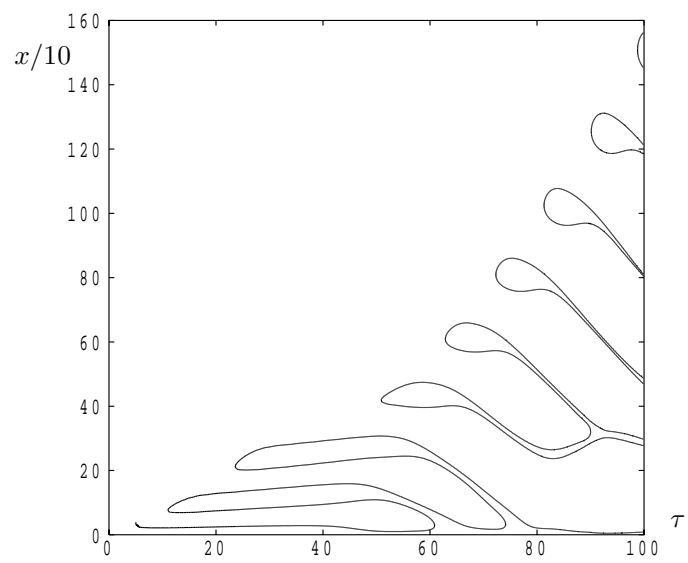

FIGURE 6f. Contours of $Q=0$ when $A=1$. 
But as $A$ increases further, nonlinear effects become more noticeable. In Fig. $6 \mathrm{c}$ and onwards, there is a distinctive drawing together of pairs of $Q=0$ contours at larger values of $\tau$, and this phenomenon occurs earlier as $A$ increases. This drawing together corresponds to having a relatively hot cell above the surface which is being squeezed upwards by two neighbouring cold cells. The pattern of streamlines takes a distinctive triangular shape similar to those seen in [3], and it heralds subharmonic instabilities.

A related feature of nonlinearity is that the hot cells rise further from the surface than for linear disturbances, and form embryonic plumes. The upward flow within these plumes must entrain fluid equally from both sides in order to at least maintain their strength, but this isn't always possible. Therefore a plume will migrate towards its nearer neighbour in an attempt to equalise the rate of entrainment; this provides a mechanism for the plumes to merge and to reduce the wavenumber. Such an event may be seen in the bottom right corners of Figs. $6 \mathrm{~d}$ and $6 \mathrm{e}$, which is where the cell spacing is narrowest. There is also an internal plume-merging event in Fig. $6 \mathrm{f}$ at $\tau=90$ and $x \simeq 340$.

This behaviour also arises in the detailed simulations of Riaz et al. [12], who considered an identical configuration to the present one, but where the initial disturbances were random. In a different context, Brambles and Rees [13] found that the centreline of a plume formed by a line source of heat near to a vertical wall traces out a curved path towards the wall. In addition, plumes caused by two parallel line sources merge into one. The numerical study of Banu et al. [14] of two dimensional convection inside a cavity with uniform heat generation describes plume merging events. When the cavity is shallow and the Darcy-Rayleigh number is relatively large, there exists a thin, but thermoconvectively unstable layer near the upper surface. Instabilities manifest themselves as travelling waves from the centre to the sidewall, and such waves often merge together.

Finally, Fig. 6 shows that the nonlinearity actually serves to delay cell formation and hence the rate of spread of the convection pattern is inhibited.

\section{CONCLUSIONS}

In this paper we have made the first steps in understanding how an isolated disturbance propagates within an unsteady diffusive boundary layer. In the linear regime the pattern spreads horizontally because new cells are formed on the edge of the pattern, and cells widen with time. These phenomena have been explained within the context of the linear stability of monochromatic modes. When $A$ is large, we have found typically nonlinear behaviour: strong inflow and outflow effects, and cell-merging events.

\section{ACKNOWLEDGEMENTS}

A.S. would like to thank the University of Bath for a Departmental Studentship and an Overseas Research Award to support this research.

\section{REFERENCES}

1. A. Selim, D.A.S. Rees (2007) The instability of a developing thermal front in a porous medium. I Linear theory. J. Porous Media. 10: 1-15.

2. A. Selim, D.A.S. Rees (2007) The instability of a developing thermal front in a porous medium. II Nonlinear evolution. J. Porous Media. 10: 17-33.

3. A. Selim, D.A.S. Rees (2010) The instability of a developing thermal front in a porous medium. III Subharmonic instabilities. To appear in J. Porous Media.

4. D.A.S. Rees, A. Selim, J.P. Ennis-King (2008) The instability of unsteady boundary layers in porous media. Emerging Topics in Heat and Mass Transfer in Porous Media ed. P.Vadasz. (Springer), pp. 85110.

5. D.A.S. Rees, A.P. Bassom (1993) The nonlinear nonparallel wave instability of free convection induced by a horizontal heated surface in fluidsaturated porous media. J. Fluid Mech.. 253: 267296.

6. D.A.S. Rees (1993) A numerical investigation of the nonlinear wave stability of vertical thermal boundary layer flow in a porous medium. J. Appl. Math. Phys. (Z.A.M.P.). 44: 306-313.

7. D.A.S. Rees (2003) Vertical free convective boundary-layer flow in a porous medium using a thermal nonequilibrium model: elliptical effects. $J$. Appl. Math. Phys. (Z.A.M.P.). 54: 437-448.

8. D.A.S. Rees, D.A. Nield, A.V. Kuznetsov (2008) Vertical free convective boundary-layer flow in a bidisperse porous medium. Trans.A.S.M.E. J. Heat Transfer. 130: 092601,1-9.

9. A.V. Getling (1992) Convective-front propagation and wavenumber selection. Physica D. 55: 121134.

10. W. van Sarloos (2003) Front propagation into unstable states. Physics reports - Review section of Physics Letters. 386: 29-222.

11. J. Kockelkoren, C. Storm, W. van Saarloos (2003) Evidence for slow velocity relaxation in front propagation in Rayleigh-Bénard convection. Physica D - Nonlinear Phenomena. 174: 168-175.

12. A. Riaz, M. Hesse, H.A. Tchelepi, F.M. Orr (2006) Onset of convection in a gravitationally unstable diffusive boundary layer in porous media. J. Fluid Mech.. 548: 87-111.

13. O.J. Brambles, D.A.S. Rees (2007) Curved free convection plume paths in porous media. Időjárás. $Q$. J. Hung. Met. Service. 111: 109-121.

14. N. Banu, D.A.S. Rees, I.Pop (1998) Steady and unsteady convection in rectangular porous cavities with internal heat generation. Proc. 11th Int. Heat Transfer Conf. (Kyongju, Korea) 4: 375-380. 